\title{
Hybrid Simulation Using SAHISim Framework
}

\section{[A Hybrid Distributed Simulation Framework Using Waveform Relaxation Method Implemented Over the HLA and the Functional Mock-up Interface]}

\author{
Muhammad Usman \\ Awais \\ Austrian Institute of \\ Technology \\ Giefinggasse 2 \\ 1210 Vienna, Austria \\ Muhammad.Awais.fl \\ @ait.ac.at
}

\author{
Wolfgang Gawlik \\ Vienna University of \\ Technology \\ Gusshausstrasse 25/370-1 \\ 1040 Vienna, Austria \\ wolfgang.gawlik \\ @tuwien.ac.at
}

\author{
Peter Palensky \\ Delft University of Technology \\ Department of Electrical \\ Sustainable Energy \\ P.Palensky@tudelft.nl
}

\author{
Gregor De-Cillia \\ Austrian Institute of \\ Technology \\ Giefinggasse 2 \\ 1210 Vienna, Austria \\ Gregor.DeCillia.fl \\ @ait.ac.at
}

\begin{abstract}
Hybrid systems such as Cyber Physical Systems (CPS) are becoming more important with time. Apart from CPS there are many hybrid systems in nature. To perform a simulation based analysis of a hybrid system, a simulation framework is presented, named SAHISim. It is based on the most popular simulation interoperability standards, i.e. High Level Architecture (HLA) and Functional Mock-up Interface (FMI). Being a distributed architecture it is able to execute on cluster, cloud and other distributed topologies. Moreover, as it is based on standards so it allows many different simulation packages to interoperate, making it a flexible and robust solution for simulation based analysis. The underlying algorithm which enables the synchronization of different simulation components is discussed in detail. A test example is presented, whose results are compared to a monolithic simulation of the same model for verification of results.
\end{abstract}

\section{Categories and Subject Descriptors}

I.6.0 [Simulation and Modeling]: General

\section{Keywords}

High Level Architecture, Functional Mock-up Interface, Modelica, OpenModelica, Simulation Interoperability, Hybrid Simulation, Co-simulation, Heterogeneous Simulation

\section{INTRODUCTION}

Standardized Architecture for Hybrid Interoperability of Simulations (SAHISim) is a newly proposed framework. It supports distributed simulation by conforming to High Level Architecture (HLA) [7]. It is flexible and robust; because individual federates can be FMI [2] components (Functional Mock-up Units (FMUs)). The purpose of developing SAHISim is to provide simulation engineers a platform which

- Supports interoperability among different simulation packages.

- Is able to add parallelism through distribution

- Supports hybrid simulation

Simulation interoperability is becoming very important in modern research and analysis. Because there are so many specialized simulation packages for different types of systems, it is appealing to use specialized simulation packages for any two or more domains and couple them to analyze results of the system. This saves considerable effort of developing and testing a new simulation package which could cover many domains. This is similar to the re-usability trend humanity has enacted in every other domain.

Interoperability among simulations becomes more vital when it can offer parallelism through distribution. Because simulation is established as a good method of verification, large scale simulations are becoming appealing in industry to verify policies and designs. Large scale simulations require large amount of computing resources. Running large scale simulations on monolithic platforms can result into resource starvation. By distributing the execution of different subsystems onto different machines, parallel execution can improve the overall performance. It can be used to avoid resource starvation by adding more remote resources. 
Simulation of hybrid systems has been a major challenge for researchers in the field of modeling and simulation. It has become even more important due to intermix of Information and Communication Technology (ICT) with other technologies. Due to the discrete aspects of ICT, its coupling with any continuous physical system leads to a hybrid system. Interoperability becomes essential in this scenario, because like other domains ICT has its own breed of specialized simulation packages. Coupling an ICT oriented simulation package with a simulation package for a physical phenomenon requires both interoperability and hybridization of the simulation.

In next section previous approaches to hybrid system simulators are discussed. Then the simulation algorithm implemented in SAHISim framework is discussed in detail in section 3. After understanding the underlying working of the framework, results of a test example are presented in section 4 , followed by a brief conclusion.

In the presented paper, major focus is on developing a synchronization algorithm for hybrid systems. The algorithm, with a little effort, can be adapted to any platform, in that case though, it will lose some functionality. For example, if it is implemented without the HLA then it will lose the ability to create simulation on distributed environments. If something else is placed instead of HLA then it will lose its standardized format. FMUs are the basic simulation components of the framework. If FMUs are replaced by some other components then again it will lose its standardized format. Nevertheless, the presented algorithm serves well to facilitate a distributed hybrid simulation.

\section{RELATED WORK}

Due to introduction of ICT into power grids management, it has become vital to simulate power grids in conjunction with ICT infrastructure. In recent past there have been quite a number of efforts to couple ICT network simulators with power system simulators [11] to simulate cyber physical energy systems, or to simulate smart grids [14]. Most smart grid simulators do not try to couple more than one continuous systems. Only a continuous power system simulator is coupled with a discrete network simulator. In this case the system formed does not have any algebraic relationship among simulation components, which makes things much easier and manageable.

When a simulation has more than one continuous simulators in the federation, things become much more complex. Many real world scenarios require such a simulation. For example, a complex energy system simulation may also need to couple thermal energy simulator along with power and network simulators. In case of more than one continuous simulators, coupling may form a Differential Algebraic Equation (DAE) with index $\geq 1$. When such tightly coupled systems are integrated over time domain, explicit methods normally do not produce good results [6]. The presented algorithm can deal with any number of continuous or discrete event simulators coupled with each other.

There have been other attempts to orchestrate hybrid simulations (alternatively called as "heterogeneous simulation") for example, Discrete EVent Specification (DEVS). DEVS provides a systematic way to convert a continuous simulator into a discrete event simulator [4]. In this way there is no difference left in coupled continuous or discrete event simulation. Although, there are still some questions related to its stability [3]. The biggest disadvantage of DEVS is its lack of interoperability with other simulation paradigms. In order to orchestrate a DEVS based federation all simulation federates must conform to DEVS specification.

Ptolemy II is another solution which aims to provide hybrid simulation platform. According to some [9] the Ptolemy Discrete Event Model Specification (PDEMS) can be considered equal to DEVS. Ptolemy II is supplemented by support for FMI [12], which makes it more usable in terms of interoperability than DEVS. However, Ptolemy II is monolithic in nature and there is still a lot of work to be done to make it distributed in nature. Secondly, there are some underlying restrictions imposed by Ptolemy II kernel which makes it difficult to implement new synchronization algorithms in Ptolemy II. Lasnier et al. [8] have used federates implemented using Ptolemy with HLA. They use the Discrete Event (DE) director of Ptolemy for federate implementation, which cannot be better than the discretization technique proposed by DEVS experts.

Techniques like [8] and [13] use nonzero lookahead based timing services of the HLA. They can be considered as explicit and hence less stable [6]. They are explicit, because for a DAE predicting "precisely" when next event will occur is not possible without actually integrating the DAE. In case of a tightly coupled system of DAEs modeled as separate federates, it is impossible to know the exact time of an event of a federate independently. In such a situation the guess work involved in setting the lookahead value and length of time step makes the solution even more erroneous.

\section{DESCRIPTION OF THE ALGORITHM}

The underlying execution of simulation using SAHISim framework is governed by a master-slave algorithm. The master algorithm works like an orchestrator. It directs the slaves to perform some action by sending them commands. The slave algorithm is a state machine, whose states are changed based on the commands received from the master. The actual numerical integration is performed at salve only, but master has to check for convergence. To check convergence, master needs to know the values of state variables at each iteration. Based on the values of state variables, master also changes the step size. Handling of discrete event is also dependent on the master. The slave which experiences a discrete event informs the master about the event, and then master orchestrates the method to handle it.

\subsection{The master algorithm}

The algorithm shown in figure 1a describes the flow of master. The master algorithm has only one instance for one simulation federation. There is no limitation on the number of slaves.

Important to mention is the decision state "Is discrete event?" in figure 1a. This is a point where the algorithm gets into a specialized flow of discrete event handling. If there is no discrete event in the system, which means that all the subsystems are continuous then this path is never executed. 


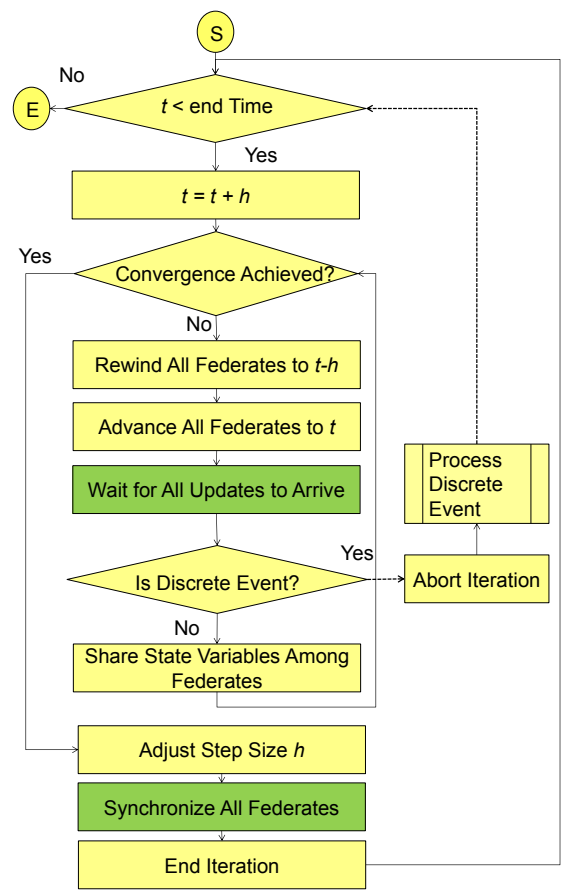

(a) Main flow of the master algorithm, (b) Discrete event processing. Here $h$ highlighted in green are steps which en- represents the step size, and $t$ represents force all the slaves to get synchronized. the time.
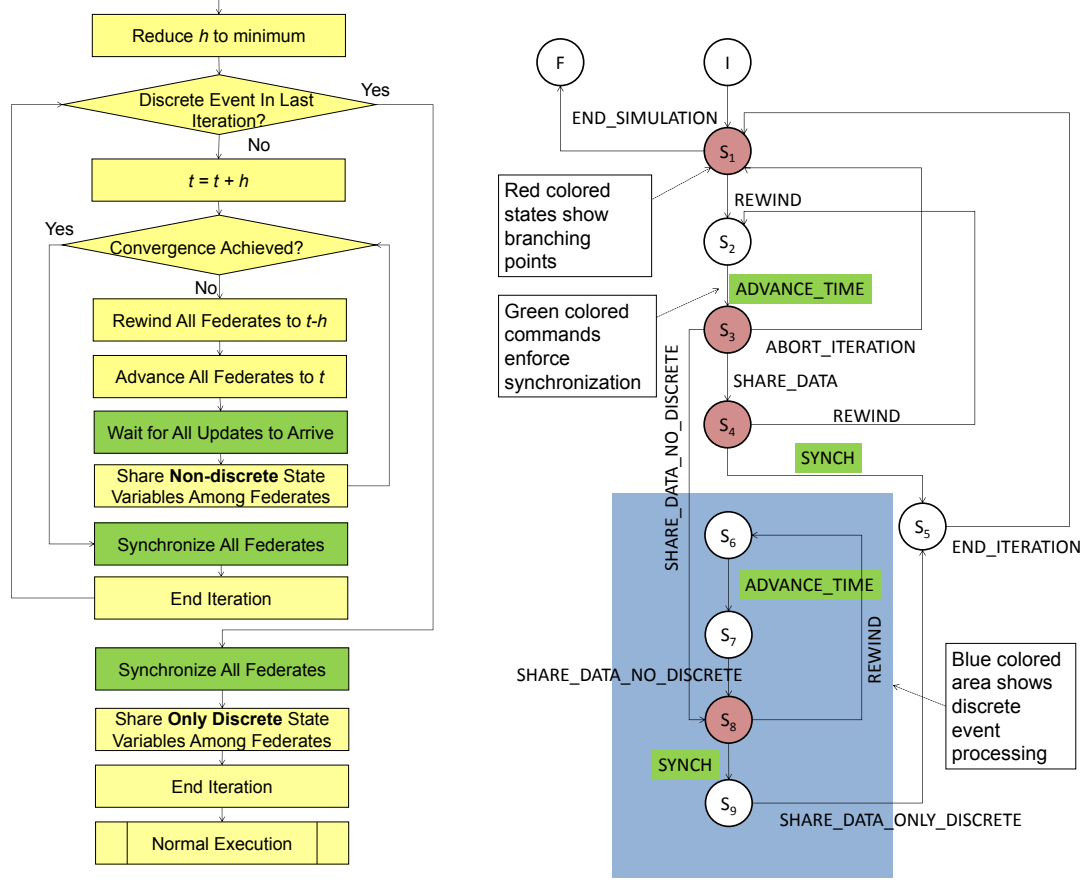

(c) State machine of the slave.
So the algorithm works fine for both hybrid and continuous systems.

\subsection{Slave State Machine}

The slave works like a state machine. Figure 1c shows that how different commands take slave from one state to another. State based execution is essential for a slave to avoid ambiguities. State based execution ensures that a slave will always keep following the correct path. Highlighted in red are the states which have a branching factor greater than 1. From these states a slave may go in wrong direction of execution if there was no method of synchronization.

\subsection{Working of Algorithm}

The mathematical motivation for the presented algorithm comes from the Waveform Relaxation (WR) algorithm [10]. The WR algorithm is only focused on the continuous simulations and does not mention handling of discrete events. The presented work augments the WR algorithm by introducing the logic of handling discrete events. The WR algorithm is based on the idea of Banach spaces, or fixed point iteration [5]. The idea is, if there is a system of Ordinary Differential Equations (ODEs), then if the state based representations of ODEs are separated in a way that performing a WR iteration is contractive in nature, then the system of equations is guaranteed to converge at each time step [10]. From one time step to another WR iteration is performed repeatedly, and at each time step the system converges at a single point, called the fixed point. In order to have a successful separation of ODEs few guideline are mentioned in the original work [10]. The WR iteration is a very simple phenomenon itself. Figure 2 shows the execution cycle of a WR iteration. All the ODE based subsystems are evaluated at time

$t_{n+1}$. The output of each subsystem is propagated to other dependent subsystems. The output values of one subsystem become the inputs for others. The time is rewound to $t_{n}$ and now the state variables of each subsystem are evaluated again at $t_{n+1}$. Continuing in this way, after few iterations the fixed point is reached, then the same procedure is used for $t_{n+2}, t_{n+3}$, so on and so forth. Here $t_{n}$ is termed as a "communication point".

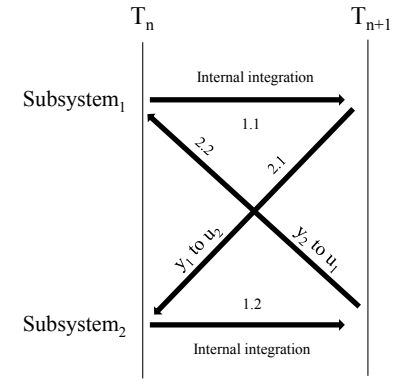

\subsection{Explanation of Commands}

The slave algorithm only follows the commands, so it is very important to understand their meaning. The actions taken to follow the commands are straight forward. The "REWIND" command takes the FMU back in time. This means that the time and state variables of the FMU are set to the values converged at the end of previous communication step. The "ADVANCE_TIME" is the command where actual integration of the subsystem (here FMU) is done. The command takes a parameter, which is the "time" to which an FMU should integrate. This is the time $t_{n}$ mentioned earlier at which repeated WR communications 
occur for convergence. The "ABORT_ITERATION" command is a level stronger than "REWIND" command. The "ABORT_ITERATION" command not only goes back in terms of time and state variables, but it also reverts to inputs valid at the end of previous successful communication step.

The "SYNCH" command is just used for the synchronization purpose. On receiving "SYNCH" command a slave sends its time to the master. Immediately after sending "SYNCH" command the master goes into an infinite loop until it receives updates from all the FMUs. It is a subject of detailed discussion how positioning this command correctly ensures correct order of execution. The proof is omitted for the sake of brevity.

The "SHARE_DATA" command asks all FMUs to share state variables. The sharing happens with the help of object management services of HLA. As a rule of thumb one state variable must be published by only one federate, but it can be subscribed by many.

The working of commands "SHARE_DATA_NO_DISCRETE" and "SHARE_DATA_ONLY_DISCRETE" is just the same as "SHARE_DATA", but there are semantic differences. Both of these commands are only used when processing a discrete event. The "SHARE_DATA_NO_DISCRETE" initiates a change in the slave state machine. On receiving this command it is known that there is a discrete event processing going on. It asks salves to share all states except the discrete ones. The 'SHARE_DATA_ONLY_DISCRETE' command is executed only once in an iteration. In result the internal behavioral change necessary to occur due to a discrete event is accomplished at once.

The command "END_ITERATION" is issued when the convergence is achieved for one communication step. On receiving this command an FMU closes the internal integration step and prepares for the next communication step.

\subsection{Processing Discrete Event}

First of all, this should be kept in mind that before processing the discrete event the iteration where the discrete event was detected has already been aborted. Abortion of the iteration means that all the FMUs go back to the state (including input variables) where they were at the end of the last communication step. The easiest way to process the discrete event is to reduce the step size to minimum and keep on progressing the simulation. Decreasing the step size to minimum ascertains that the precise time of event cannot be missed by far, which reduces the chances of error propagation. The simulation with minimum step size continues until the discrete event has occurred again. After the iteration in which discrete event occurs, the master switches back to the normal mode of execution.

\subsection{HLA Timing Services}

Time Advance Request (TAR) and Time Advance Request Available (TARA) services are used in collaboration at both master and slave level. The step size is calculated by the master at the end of each communication point. Before convergence TARA service is used repeatedly by the master and the slaves, to get the updates. Once the convergence is achieved and "END_ITERATION" command is issued, the TAR service is invoked by the master and slaves to close the episode. In this way any discrete event simulator using the same services will not have any problem in co-simulation.

For clarity it is important to mention that when a federate issues TARA request with time $t_{0}$ then it announces that it may send more updates at the same time $t_{0}$. Similarly, it is ready to accept more updates from other federates at $t_{0}$. Once the master decides that it is done with the iteration and there are no more updates needed, it issues "END_ITERATION" command with TAR at time $t_{0}$, subsequently all slaves also issue TAR. After issuing TAR any federate can send an update only at a time $t_{0}+\epsilon$ for any $\epsilon>0$.

\subsection{Communication Step Size Control}

Step size control offers many advantages in any numerical integration algorithm. Implemented correctly, it can significantly enhance the performance of the algorithm. Here too, the communication step size control offers many advantages. Most importantly, in a distributed simulation more communication steps mean more communication, which means lesser performance. So increasing the communication step size to the maximum where the solution remains valid is very beneficial.

Looking at the figure 2, it is easy to understand that separating the ODEs means that some or all of the sate variables in subsystems are going to grow independent of partial derivatives of each other. Mathematically speaking, suppose there is a system given in equation 1

$$
\dot{y}=f(y, p)
$$

The state vector $y$ contains $n$ state variables $y=y_{1}, y_{2}, y_{3}, \ldots, y_{n}$. To perform the numerical integration of the system, if an implicit method is used, then the Jacobian of the system will be $n \times n$ matrix, containing partial derivatives of all the state variables with respect to each of them. Partitioning the system in two (equation 2) means that the Jacobian of each subsystem is also reduced to some degree. If $\hat{y}=y_{1}, y_{2}, y_{3}, \ldots, y_{i}$ and $\tilde{y}=$ $y_{i+1}, y_{i+2}, y_{i+3}, \ldots, y_{n}$, then this means that state variables in $\hat{y}$ are being evaluated without their partial derivatives with respect to $y_{i+1}, y_{i+2}, y_{i+3}, \ldots, y_{n}$. Similar is the case of $\tilde{y}$. This causes divergence in the solution. If the divergence remains in the realm where the system remains defined then it is possible to recover the error through fixed point iteration. If not, then this means that the gap between two communication steps is too large.

$$
\begin{aligned}
\dot{\hat{y}} & =\hat{f}(\hat{y}, \hat{p}) \\
\dot{\tilde{y}} & =\tilde{f}(\tilde{y}, \tilde{p})
\end{aligned}
$$

Following the idea of divergence, apart from error tolerance, there is an additional parameter introduced, which is called as "divergence tolerance" $t o l_{d}$. This is tolerance for the error caused by divergence. If the state variable vector, as a result of initial guess at the start of WR iteration, is $y_{i}$, and at the end of WR iteration after convergence is $y_{f}$, then the error $e_{d}$ caused by divergence is given in equation 3 .

$$
e_{d}=\left\|y_{f}-y_{i}\right\|_{2}
$$




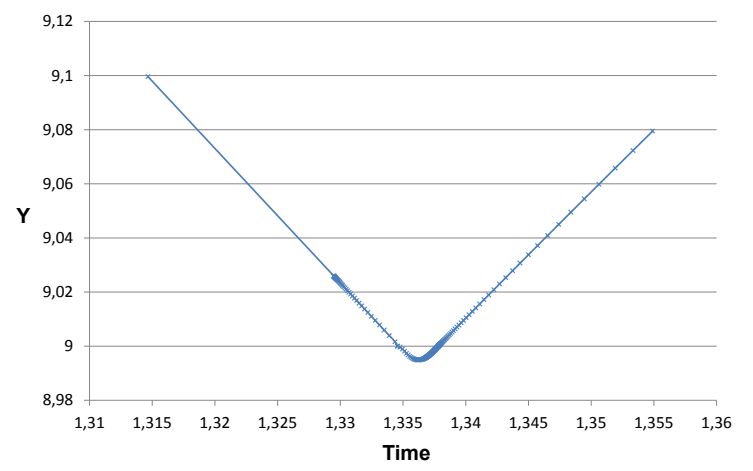

Figure 3: Variation of communication step size during processing of discrete event.

At the end of each WR iteration the communication step size is either increased or decreased by some percent, based on the fact that $e_{d}+\tau_{0}\left\|y_{f}-y_{i}\right\|_{\max }<$ tol $_{d}$ or $e_{d}+\tau_{0}\left\|y_{f}-y_{i}\right\|_{\max }>$ tol $_{d}$. Here $\tau_{0}$ is a small positive value used for normalization. As mentioned in section 3.5, during processing of discrete event, the communication step size is intermediately reduced to minimum. After the discrete event, communication step size takes some time to recover its value. At that moment the mechanics of communication step size control become evident. Figure 3 shows the phenomenon by zooming into that situation.

\section{TEST CASE}

To check the correctness of algorithm a test system is used for simulation. It is first simulated using the OpenModelica $^{1}$. The results are compared with the SAHISim algorithm presented earlier. The system is very popular hybrid system i.e. a ball being dropped from a height on stairs, namely a "bouncing ball on stairs". The system is given by the system of equations 4 . The discrete part is given by algorithm 1

$$
\begin{aligned}
\dot{x} & =v_{x} \\
\dot{y} & =v_{y} \\
\dot{v_{x}} & =-c_{0} v_{x} \\
\dot{v_{y}} & =-g-c_{1} v_{y}-\operatorname{contact}\left((y-\operatorname{stair}) c_{2}+c_{3} v_{y}\right)
\end{aligned}
$$

Here $g$ is the gravitational constant, while $c_{0}, c_{1}, c_{2}$ and $c_{3}$ are few constants facilitating the phenomena of friction, air resistance, damping and mass of the ball. The variables stair and contact represent discrete variables. The variable contact shows that the ball is in contact with the floor or not. When contact $=1$ the system shifts its behavior immediately at that point. This is what is called as "behavioral state change". The variable stair shows that on which step of the stair ball is currently bouncing. Initially, its value is $\mathcal{N}$ used in algorithm 1.

Figure 4 shows how different FMUs are associated to each other, via their state variables. All the subsystems are in form of FMUs, which shows the ability of SAHISim framework to generically host any simulation comprising of components conforming to FMI. Figure 5 shows the simulation results of all continuous state variables, as simulated

\footnotetext{
${ }^{1}$ https://www.openmodelica.org/
}
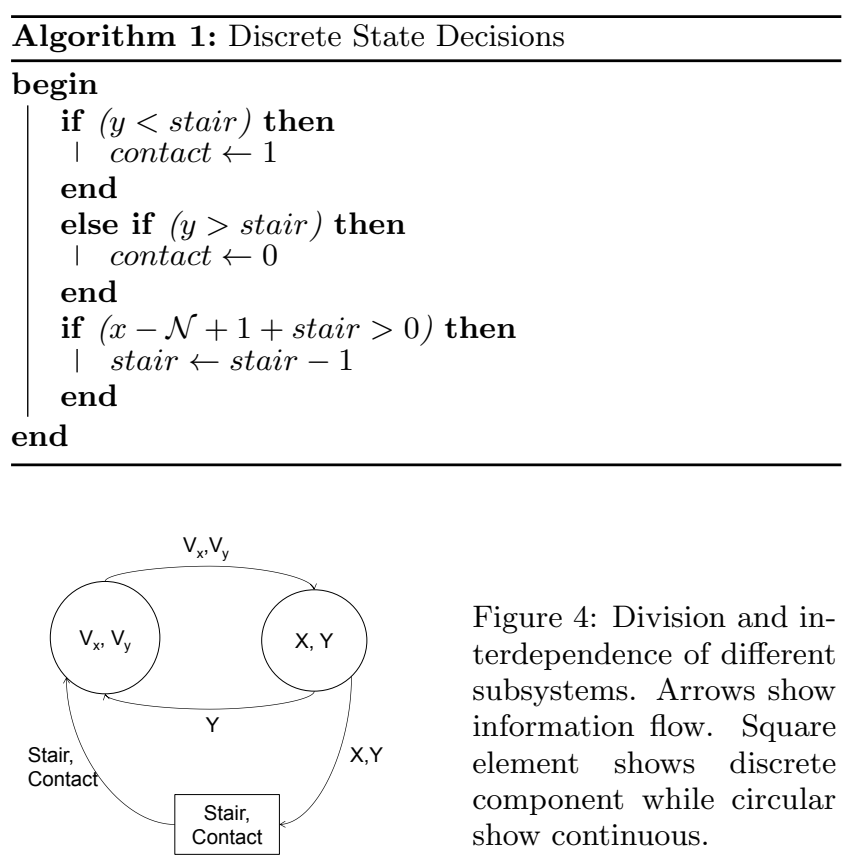

Figure 4: Division and interdependence of different subsystems. Arrows show information flow. Square element shows discrete component while circular show continuous.

by OpenModelica. The results of SAHISim simulation are shown in figures 5 . It is clear that the over all behaviors of both systems are similar, with little differences.

For the presented run, the value of "divergence tolerance" was $t_{\text {ol }}=1 \times 10^{-3}$. Although, the value is relatively large, using a smaller value makes results more accurate, but that causes more communication steps and hence performance deteriorates.

\section{CONCLUSION}

The paper presents Standardized Architecture for Hybrid Interoperability of Simulations (SAHISim). In depth discussion on the working of synchronization algorithm and data sharing using HLA services is presented. The use of SAHISim framework is very easy, it abstracts away tedious configuration details of HLA and makes it very easy for the user to orchestrate a federated simulation. A generic thin layer is implemented to enable the use of FMUs as federates of the simulation federation. Enabling FMI makes the solution very flexible because there are more than forty simulation packages ${ }^{2}$ either supporting or planning to support FMI. Currently only FMI 1.0 compliant components are supported by SAHISim. The main reason is that FMI 2.0 is not yet supported by open source simulation packages.

The algorithm used by SAHISim is discussed in detail. The use of presented algorithm is not tied to the use of SAHISim. It can be used in many different ways, independent of SAHISim framework. A test example comparing the results with a monolithic simulation package (OpenModelica) shows the correctness of the results. The results are promising and inspire further development. There are few problems still to be tackled. Currently, individual subsystems are integrated using self-developed solvers. In order to use industry level solvers it is important to make few changes in them. The

\footnotetext{
${ }^{2}$ https://www.fmi-standard.org/tools
} 

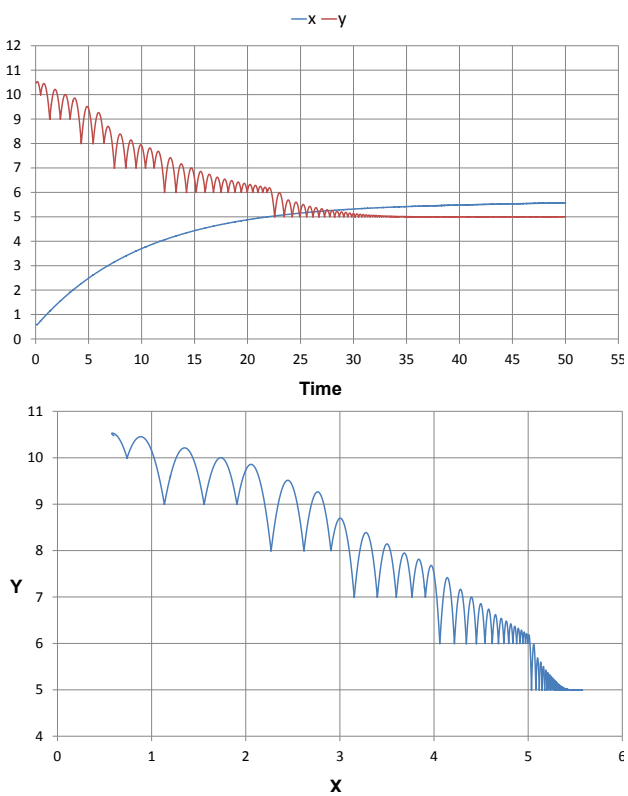
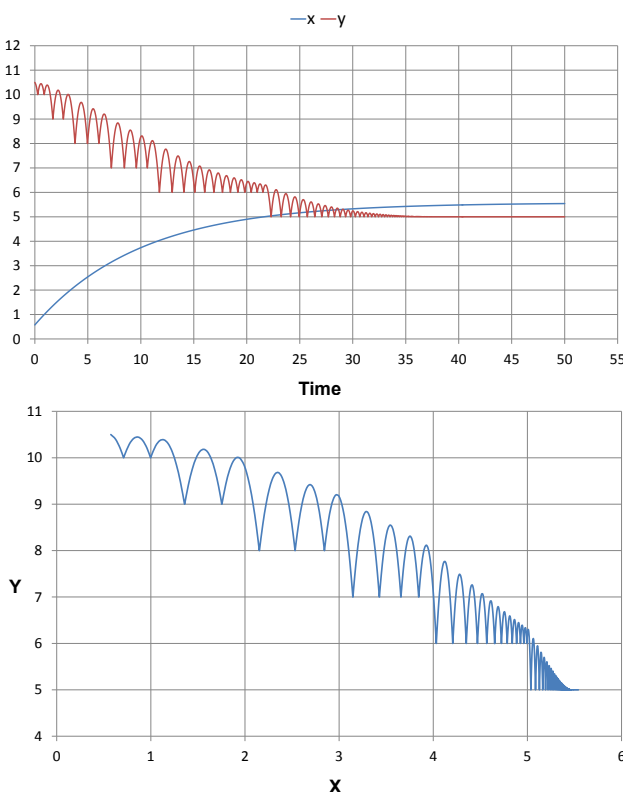

Figure 5: On left the results from SAHISim are shown, on right are the results obtained from OpenModelica.

most important change is to be able to rewind the solver to the previously calculated state. If professional solvers can incorporate this change, then it will enable their use in SAHISim framework, making it even more robust.

\section{REFERENCES}

[1] M. Awais, P. Palensky, A. Elsheikh, E. Widl, and M. Stifter. The high level architecture RTI as a master to the functional mock-up interface components. In International Workshop on Cyber-Physical System (CPS) and its Computing and Networking Design (ICNC 2013), pages 315-320, January 2013.

[2] T. Blochwitz, M. Otter, M. Arnold, C. Bausch, C. Clauß, et al. The functional mockup interface for tool independent exchange of simulation models. In Modelica'2011 Conference, pages 20-22, March 2011.

[3] F. E. Cellier and E. Kofman. Continuous system simulation. Springer US, 2006.

[4] M. D'Abreu and G. Wainer. Models for continuous and hybrid system simulation. In Simulation Conference, 2003. Proceedings of the 2003 Winter, volume 1, pages 641-649 Vol.1, Dec 2003.

[5] V. Istrc et al. Fixed point theory: an introduction, volume 7 of Mathematics and Its Applications. Springer, 1981.

[6] R. Kübler and W. Schiehlen. Two methods of simulator coupling. Mathematical and Computer Modelling of Dynamical Systems, 6(2):93-113, 2000.

[7] F. Kuhl, R. Weatherly, and J. Dahmann. Creating computer simulation systems: an introduction to the high level architecture. Prentice Hall PTR, 1999.

[8] G. Lasnier, J. Cardoso, P. Siron, C. Pagetti, and P. Derler. Distributed simulation of heterogeneous and real-time systems. In Proceedings of the 2013 IEEE/ACM 17th International Symposium on Distributed Simulation and Real Time Applications, pages 55-62. IEEE Computer Society, 2013.
[9] H. Y. Lee, W.-T. Kim, I.-G. Chun, W. Kang, and S.-M. Park. A formal representation of discrete event models in ptolemy ii. In Advanced Communication Technology (ICACT), 2010 The 12th International Conference on, volume 1, pages 864-869, Feb 2010.

[10] E. Lelarasmee, A. E. Ruehli, and A. L. Sangiovanni-Vincentelli. The waveform relaxation method for time-domain analysis of large scale integrated circuits. IEEE Transactions on Computer-Aided Design of Integrated Circuits and Systems, 1(3):131-145, 1982.

[11] K. Mets, J. Ojea, and C. Develder. Combining power and communication network simulation for cost-effective smart grid analysis. Communications Surveys \& Tutorials, Issue: 99:xx, 2014.

[12] W. Müller and E. Widl. Linking FMI-based components with discrete event systems. In 2013 IEEE International Systems Conference Proceedings, page 5 pages. IEEE Conference Publications, 2013.

[13] H. Neema, J. Gohl, Z. Lattmann, J. Sztipanovits, G. Karsai, S. Neema, T. Bapty, J. Batteh, H. Tummescheit, and C. Sureshkumar. Model-based integration platform for fmi co-simulation and heterogeneous simulations of cyber-physical systems. In 10th International Modelica Conference, pages 10-12, 2014.

[14] P. Palensky and F. Kupzog. Smart grids. Annual Review of Environment and Resources, 38(1):201-226, 2013. 